\title{
Predictors of Treatment Attrition Among an Outpatient Clinic Sample of Youths With Clinically Significant Anxiety
}

\author{
Araceli Gonzalez $\cdot$ V. Robin Weersing • \\ Erin M. Warnick • Lawrence D. Scahill • \\ Joseph L. Woolston
}

Published online: 26 October 2010

(c) The Author(s) 2010. This article is published with open access at Springerlink.com

\begin{abstract}
Predictors of treatment attrition were examined in a sample of 197 youths (ages 5-18) with clinicallysignificant symptoms of anxiety seeking psychotherapy services at a community-based outpatient mental health clinic (OMHC). Two related definitions of attrition were considered: (a) clinician-rated dropout (CR), and (b) CR dropout qualified by phase of treatment (pre, early, or late phases) (PT). Across both definitions, rates of attrition in the OMHC sample were higher than those for anxious youths treated in randomized controlled trials, and comorbid depression symptoms predicted dropout, with a higher rate of depressed youths dropping out later in treatment (after 6 sessions). Using the PT definition, minority status also predicted attrition, with more AfricanAmerican youths lost pre-treatment. Other demographic (age, gender, single parent status) and clinical (externalizing symptoms, anxiety severity) characteristics were not significantly associated with attrition using either definition. Implications for services for anxious youths in public service settings are discussed. Results highlight the important role of comorbid depression in the treatment of anxious youth and the potential value of targeted retention
\end{abstract}

A. Gonzalez $(\bowtie) \cdot$ V. R. Weersing

San Diego State University/University of California, Joint

Doctoral Program in Clinical Psychology, 6363 Alvarado Court,

Suite 103, San Diego, CA 92120-4913, USA

e-mail: arg001@ucsd.edu

E. M. Warnick · J. L. Woolston

Outpatient Psychiatric Clinic for Children, Yale Child Study

Center, 40 Temple Street Suite 7C, New Haven, CT 06510, USA

L. D. Scahill

Child Psychiatry, Yale Child Study Center and Yale School

of Nursing, 40 Temple Street, Suite 7C, New Haven,

CT 06510, USA efforts for ethnic minority families early in the treatment process.

Keywords Treatment attrition - Child anxiety · Adolescent anxiety · Outpatient psychiatry .

Community mental health services

Approximately half of all adults (Garfield 1994; Wierzbicki and Pekarik 1993) drop out of outpatient therapy; the range is wider for youths, with estimates that approximately $35-75 \%$ of children terminate services before the provider would agree that it is appropriate (Kazdin 1990; Miller et al. 2008). These findings suggest that many ostensibly "treated" individuals in need of mental health services receive less than an adequate dose of care. Attrition results in inefficient use of services in the present (Armbruster and Kazdin 1994) and may create additional costs in the future as individuals with unresolved symptoms return to care in a quick churning of short, under-dosed episodes (Kazdin 1990).

The majority of research on attrition has focused on adult populations, and findings have spurred the development of brief engagement interventions designed to address practical and psychological barriers to treatment participation (see Walitzer et al. 1999). Initial studies of engagement in child mental health services and the use of adjunct services for attrition prevention have shown promise (May et al. 2007; McKay et al. 1998; Nock and Kazdin 2005); however, work on engagement and reducing attrition from youth mental health services remains scarce and identification of risk factors for attrition is an important prerequisite to such interventions. The relatively small body of work on attrition from youth psychotherapy has yielded inconsistent findings and has been dominated by studies of children and 
adolescents with disruptive behavior disorders (e.g., Armbruster and Kazdin 1994; Friars and Mellor 2009; Gross et al. 2001; Kazdin et al. 1993; Kazdin and Mazurick 1994; Kazdin and Wassell 1998; Luk et al. 2001) or general clinical samples (Attride-Stirling et al. 2004; Miller et al. 2008; Pelkonen et al. 2000). This is not surprising, as parents more often refer their children for treatment of externalizing than internalizing problems (Sayal et al. 2002; Wu et al. 1999). In the community, however, there is evidence that, as a class, anxiety is one of the most common youth mental health disorders (Cartwright-Hatton et al. 2006; Costello et al. 2003; Kashani and Orvaschel 1990). Untreated, early-onset anxiety often continues into adulthood (Dadds et al. 1999; Keller et al. 1992), and predicts academic underachievement, substance dependence (Woodward and Fergusson 2001), and the development of depression and conduct disorder (Bittner et al. 2007).

Given the persistent course of anxiety, retention of these youths in treatment for an adequate dose of care seems an important public health priority, and research is needed to identify reliable risk factors of treatment non-completion in youths with clinically significant anxiety. Youths with anxiety are most often served in school or communitybased outpatient mental health settings (Chavira et al. 2009), yet most of what is known regarding attrition among anxious youths is born from samples receiving treatment within the context of specialty clinics or randomized control trials (RCTs). To date, two studies have examined attrition among clinically anxious youths. Both found that a substantial portion of youths do not complete treatment (23\%), however, characteristics of completers and noncompleters were not consistent across these investigations. One study found that among anxious youth receiving cognitive-behavioral therapy (CBT) in a university specialty outpatient clinic, treatment dropouts were more likely to live in a single parent household, be an ethnic minority, and report lower anxiety at baseline than youths who completed treatment (Kendall and Sugarman 1997). By contrast, a subsequent study in a different research clinic produced no statistically significant differences between completers and noncompleters of CBT for phobias and anxiety across a combined dataset from two trials (Pina et al. 2003).

Given the availability of efficacious treatments for youth anxiety (see Compton et al. 2004; Silverman et al. 2008), successful delivery of these services in non-research clinical practice appears an important next step (Emslie 2008; La Greca et al. 2009). As an intermediate step toward treatment dissemination, it seems critical to understand the current status of care for anxious youths, including length of stay and predictors of attrition and to understand these factors within the context of anxious youths as they present in community settings. While previous attrition findings provide a useful springboard for selecting a priori predictors of attrition among anxious youths, it is reasonable to question whether findings from these samples are directly applicable to youths served in public sectors of care due to significant differences between the samples of youths and types of treatments received in usual care versus clinical research.

While RCTs strive to include demographically and clinically diverse youths with some success, there remain substantial differences between RCT samples and youths who attend outpatient mental health centers (OMHCs). In OMHCs, anxious youth often have more severe psychiatric comorbidity, are more likely a member of an ethnic minority group, and may more likely come from lowincome, single parent families (Southam-Gerow et al. 2003). As discussed previously, these demographic factors have been associated with attrition within one university clinic data set (Kendall and Sugarman 1997) and the increased prevalence of these risk factors bodes poorly for retention of anxious youth in OMHC settings. The likely impact of heightened comorbidity on attrition in usual care settings is more difficult to predict. There is some evidence that comorbid diagnoses and symptoms do not significantly predict or moderate outcomes for anxiety treatment in the context of RCTs (see Ollendick et al. 2008). These results are promising, but generalization of these findings outside of RCT samples remains unclear. In truth, "pure" anxious youths are relatively rare, or rarely identified, in nonresearch usual care mental health settings and these children and adolescents could be characterized as clinically heterogeneous youths with clinically significant anxiety (Chavira et al. 2009). Thus, separate examination of a community-based anxious population may be highly informative.

In addition to client characteristics, researchers have highlighted numerous differences between the interventions delivered in research clinics and community clinic care (e.g., Weisz et al. 1992, 1995a, 1995b). For instance, therapy in RCTs tends to be more highly structured, problem-focused, and time-limited (Hunt and Andrews 1992; Sledge et al. 1990); by contrast, treatment in outpatient community centers tends to be unstructured and eclectic (Weersing and Weisz 2002). Further, providers in RCTs typically receive extensive training and increased supervision compared to community-based providers. In addition, while usual care is open-ended, short-term research interventions may actually provide a larger dose of therapy. The mean length of treatment for empirically tested child anxiety psychosocial interventions is approximately 12 sessions (this mean was obtained by averaging the number of treatment sessions listed for each study included in a recent review by Silverman et al. (2008) of 32 clinical trials of treatment for youth anxiety and phobias), 
which is considerably higher than the mean number of three treatment sessions attended by youth in outpatient mental health settings (Hansen et al. 2002; Harpaz-Rotem et al. 2004; McKay and Bannon 2004). Lack of manualized treatments in OMHCs adds a layer of complexity to definition of dropout, as there is no pre-set number of treatment sessions required in usual care. When attrition cannot be defined based on completion of a standard protocol, therapist judgment may provide the most valid and meaningful way to define dropout, as this approach considers the reason for and appropriateness of dropout given the course of care to date (Wierzbicki and Pekarik 1993).

Despite differences in both client and setting characteristics, virtually nothing is known about whether these observed differences translate into predictors of treatment dropout among youths with anxiety in community settings. Accordingly, in the present study we aimed to extend previous investigations of treatment dropout by (a) examining attrition rates among youths with clinically significant levels of anxiety presenting at an OMHC; and (b) identify client characteristics associated with attrition in this sample. We reviewed the child treatment literature to guide our selection of a priori predictor variables for analysis in the current study. We included variables that (a) were associated with attrition in previous research (e.g., ethnic minority status, single parent household), (b) highlighted clinical characteristics relevant to our use of a community clinic sample (e.g., comorbid externalizing symptoms), and/or (c) were theoretically linked to internalizing youth, regardless of previous significance of findings in externalizing samples (e.g., age, gender, severity of anxiety, and comorbid depression symptoms). Based on our review, we selected age, gender, family composition (single- or two-parent household), minority status (yes/no; with follow-up subgroup analyses), and symptom severity of anxiety, depression, and externalizing behavior as a priori predictors of treatment dropout. We hypothesized that attrition would be higher in this community clinic sample than in previous studies utilizing RCT samples and that minority status, living with one parent, and increased depression severity would be adversely associated with dropout.

\section{Method}

The current project was approved by institutional review boards (IRBs) at each affiliated institution.

Sample

Participants were drawn from families seeking mental health services at an urban, community-based OMHC. All intake appointments were completed between July 2004 and March 2007, and all youths attended their last appointment prior to May 2008. During this timeframe, 1,316 youths were scheduled for intake and 990 intakes were completed. Youths were excluded from the current study if they were missing data on anxiety symptoms ( $n=176)$, were in immediate crisis (e.g., actively suicidal) and completed an urgent intake assessment $(n=127)$, or if a caregiver was unable to complete standardized measures in English $(n=122)$. A total of 565 youths were screened for significant symptoms of anxiety. Youths were defined as clinically anxious if caregivers reported (a) clinically significant symptoms (total score $\geq 25$ ) on the Screen for Child Anxiety and Related Emotional Disorders (SCARED; Birmaher et al. 1999) and/or (b) a diagnosis of an anxiety disorder (generalized anxiety, separation anxiety, social anxiety, panic disorder, post-traumatic stress disorder) in the past month, with at least intermediate impairment rating in one or more areas, on the Diagnostic Interview Schedule for Children for DSM-IV (DISC-IV; Shaffer et al. 2000). To be included in the study, subjects had to have both measures completed and meet these criteria on at least one measure.

One-hundred ninety-seven youths ages 5-18 ( $M=$ 10.28 years, $\mathrm{SD}=3.34$ ) met these entry criteria and were included in analyses. The sample was $69.3 \%$ male and ethnically diverse; $49.7 \%$ Caucasian, $24.4 \%$ African American, $15.7 \%$ Hispanic/Latino, and $10.1 \%$ other. The majority of youths $(84.3 \%)$ lived with one or both of their biological parents. Sixty-seven percent of families in present sample were receiving Medicaid health coverage; the sample predominantly falls within the low- to middleclass range of socioeconomic status (SES). Parental education and occupation were not collected routinely by the clinic and were available for less than $60 \%$ of families, precluding our ability to use these variables as proxies for SES. Table 1 presents demographic characteristics for the total sample and by dropout status.

Clinical characteristics of this sample are provided in Table 2. Youths in this sample also were clinically diverse; $60.0 \%$ of cases met criteria for two or more disorders on the DISC-IV, with an average of 2.3 disorders (range = 0-6). Of the total sample, $67.0 \%$ endorsed scores above the clinical cutoff on the SCARED, and $36.5 \%$ of cases met diagnostic criteria for a current diagnosis of any anxiety disorder $1^{1}$ on caregiver report on the DISC-IV (see Table 2). Clinician diagnoses were not recorded in medical records for 45 youths, however, of the 152 youths with a

\footnotetext{
1 Anxiety not otherwise specified, specific phobia, and obsessivecompulsive disorder were not assessed in this setting, thus, it is likely that the value reported for any anxiety disorder is an underestimate of the prevalence of anxiety in this sample.
} 
Table 1 Demographic characteristics of dropouts and non-dropouts by definition of attrition

\begin{tabular}{|c|c|c|c|c|c|c|}
\hline & \multirow{3}{*}{$\begin{array}{l}\text { Total } \\
\text { Sample (\%) }\end{array}$} & \multicolumn{5}{|c|}{ Clinician-rated dropout } \\
\hline & & \multicolumn{2}{|c|}{ Definition 1: Yes/No (CR) } & \multicolumn{3}{|c|}{ Definition 2: phase of treatment (PT) } \\
\hline & & $\begin{array}{l}\text { Non-dropout } \\
(\%)\end{array}$ & $\begin{array}{l}\text { Any dropout } \\
(\%)\end{array}$ & $\begin{array}{l}\text { Pre-treatment } \\
(0-1 \text { sessions })\end{array}$ & $\begin{array}{l}\text { Early (2-6 } \\
\text { sessions) }\end{array}$ & $\begin{array}{l}\text { Late }(\geq 7 \\
\text { sessions) }\end{array}$ \\
\hline$N$ & $197(100)$ & $96(48.7)$ & $101(51.3)$ & 25 & 28 & 48 \\
\hline \multicolumn{7}{|l|}{ Demographic } \\
\hline Gender (male) & $124(62.9)$ & $64(66.7)$ & $60(59.4)$ & 17 & 11 & 32 \\
\hline Mean age (SD) & $10.28(3.33)$ & $10.13(3.43)$ & $10.43(3.26)$ & 10.72 & 9.57 & 10.78 \\
\hline Caucasian & $98(49.7)$ & $50(52.1)$ & $48(47.5)$ & $7^{\mathrm{a}}$ & 13 & 28 \\
\hline Any minority & $99(50.3)$ & $46(47.9)^{1}$ & $53(52.5)$ & $18^{1}$ & 15 & 20 \\
\hline African-American & $48(24.4)$ & $22(22.9)$ & $26(25.7)$ & $12^{\mathrm{a}, \mathrm{b}, \mathrm{c}}$ & 4 & 11 \\
\hline Hispanic/Latino & $31(15.7)$ & $14(14.6)$ & $17(16.8)$ & $5^{\mathrm{c}}$ & 8 & 4 \\
\hline Other & $20(10.1)$ & $10(10.4)$ & $10(9.9)$ & $1^{\mathrm{d}}$ & 3 & 5 \\
\hline Lives with both parents & $63(32.0)$ & $32(33.3)$ & $31(30.7)$ & 7 & 11 & 13 \\
\hline$\%$ Medicaid & $132(67.0)$ & $61(63.5)$ & $71(71.3)$ & 17 & 13 & 41 \\
\hline \multicolumn{7}{|l|}{ No. sessions attended } \\
\hline Median & 9 & 14 & 6 & 0 & 4 & 20.5 \\
\hline Range & $0-78$ & $0-74$ & $0-78$ & & & \\
\hline
\end{tabular}

Note: Groups with same letter superscripts are significantly different at $p<0.05$; same number superscripts denotes $p<0.10$

documented clinician diagnosis, 77 received at least one anxiety-related diagnosis by the clinician. ${ }^{2}$ Notably, comorbidity with a non-anxiety disorder in this sample is high. This observation is consistent with existing reports that the majority of youths served in community-based clinics have externalizing problems and underscores the critical need to understand what clinically anxious youths in this setting "look like."

\section{Setting}

The OMHC is located in New Haven, Connecticut and provides services to children and adolescents. Across all presenting problems, youths who present for treatment in this clinic are predominantly lower SES, and over half of youths have current or past involvement with child welfare services (e.g. foster care or juvenile justice system). Treatment tends to be eclectic and delivered by social workers (Walker et al. 2008); in this anxious sample, psychotherapy was delivered by 58 social workers, social work

\footnotetext{
${ }^{2}$ As a conservative test, we attempted to re-run all analyses within this subsample of youths who received a diagnosis of anxiety by clinician report $(n=77)$. Pattern of results was similar in the subsample as in our larger sample. For example, for clinician-rated dropout $(\mathrm{Y} / \mathrm{N})$, depression symptom severity increased the odds of attrition in the full model (OR $=1.11,95 \%$ CI: 0.99-1.24), though this effect lapsed into non-significance $(p=0.07)$ likely due to the smaller sample size. Nevertheless, these findings lend confidence to the pattern of findings within a sample with significant anxiety. We were unable to example predictors of Phase of Treatment dropout within this subsample given insufficient number of cases in each cell.
}

trainees, and psychology or psychiatry fellows; 68\% of cases were treated by social workers. For all youths in this sample, psychotherapy services were recommended at time of intake assessment, and no youths were referred or seen for medication management without therapy. At intake, all families participated in a systematic assessment procedure designed to comply with state reporting requirements and to characterize the patient population and services. Study consent was sought from the legal guardian of all youths as part of this procedure. In some cases, when a legal guardian was not available to consent, we obtained a waiver from the institution's IRB to access medical records.

\section{Measures}

Demographic data were collected from caregivers at the first intake visit. Clinical assessments were collected during intake, and results were provided to clinicians prior to treatment delivery. Dropout ratings were coded by clinicians at patient discharge.

The Screen for Child Anxiety and Related Emotional Disorders, parent report (SCARED-P; Birmaher et al. 1999) is a 41-item parent-report questionnaire designed to screen for elevated anxiety symptoms in children and adolescents. The SCARED has demonstrated good validity and reliability in both clinical and non-clinical samples. There is evidence that a score of 25 on the SCARED has good sensitivity and specificity for distinguishing diagnosable anxious from non-anxious youths and predicting 
Table 2 Clinical characteristics of dropouts and non-dropouts by definition of attrition (past month)

\begin{tabular}{|c|c|c|c|c|c|c|}
\hline & \multirow{3}{*}{$\begin{array}{l}\text { Total } \\
\text { Sample (\%) }\end{array}$} & \multicolumn{5}{|c|}{ Clinician-rated dropout } \\
\hline & & \multicolumn{2}{|c|}{ Definition 1: Yes/No (CR) } & \multicolumn{3}{|c|}{ Definition 2: phase of treatment (PT) } \\
\hline & & Non-dropout $(\%)$ & Any dropout $(\%)$ & $\begin{array}{l}\text { Pre-treatment } \\
(0-1 \text { sessions })\end{array}$ & $\begin{array}{l}\text { Early } \\
\text { (2-6 sessions) }\end{array}$ & $\begin{array}{l}\text { Late } \\
\text { ( } \geq 7 \text { sessions) }\end{array}$ \\
\hline$N$ & $197(100)$ & $96(48.7)$ & $101(51.3)$ & 25 & 28 & 48 \\
\hline \multicolumn{7}{|l|}{ Mean Sx scores (SD) } \\
\hline SCARED & $29.89(13.94)$ & $29.33(13.58)$ & $30.43(14.32)$ & $27.72(12.01)$ & $30.29(12.27)$ & $31.92(16.41)$ \\
\hline SMFQ & $10.45(6.49)$ & $9.50^{\mathrm{a}, \mathrm{b}}(5.86)$ & $11.37^{\mathrm{a}}(6.94)$ & $10.08(7.15)$ & $10.82(5.82)$ & $12.35(7.42)^{\mathrm{b}}$ \\
\hline SNAP-ODD & $1.72(0.89)$ & $1.68(.89)$ & $1.76(.89)$ & $1.57(0.81)$ & $1.84(0.94)$ & $1.81(0.91)$ \\
\hline \multicolumn{7}{|l|}{ Above clinical cutoff } \\
\hline SCARED & $132(67.0)$ & $64(66.7$ & $68(67.3)$ & 14 & 22 & 32 \\
\hline SMFQ & $94(47.7)$ & $40(41.7)$ & $54(53.5)$ & 11 & 11 & 32 \\
\hline SNAP-ODD & $122(61.9)$ & $60(62.5)$ & $62(61.4)$ & 13 & 18 & 31 \\
\hline \multicolumn{7}{|l|}{ DISC diagnoses } \\
\hline Any anxiety disorder & $72(36.5)$ & $36(37.5)$ & $36(36.6)$ & 10 & 12 & 14 \\
\hline Generalized & $18(9.1)$ & $8(8.3)$ & $10(9.9)$ & - & 3 & 7 \\
\hline Separation & $40(20.3)$ & $20(20.8)$ & $20(19.8)$ & 7 & 6 & 7 \\
\hline Social & $31(15.7)$ & $14(14.6)$ & $17(16.8)$ & 3 & 8 & 6 \\
\hline PTSD & $1(0.5)$ & $1(1.0)$ & - & - & - & - \\
\hline Any depressive disorder & $33(16.8)$ & $13(13.5)$ & $20(19.8)$ & 5 & 4 & 11 \\
\hline MDD & $15(7.6)$ & $5(5.2)$ & $10(9.9)$ & 1 & 2 & 7 \\
\hline Dysthymia & $18(9.1)$ & $8(8.3)$ & $10(9.9)$ & 4 & 2 & 4 \\
\hline Any externalizing disorder & $126(63.9)$ & $57(59.4)$ & $69(68.3)$ & 15 & 18 & 36 \\
\hline ADHD & $107(54.3)$ & $53(55.2)$ & $54(53.5)$ & 13 & 14 & 27 \\
\hline ODD & $120(60.9)$ & $53(55.2)$ & $67(66.3)$ & 15 & 17 & 35 \\
\hline $\mathrm{CD}$ & $51(25.9)$ & $26(27.1)$ & $25(24.8)$ & 2 & 10 & 13 \\
\hline
\end{tabular}

Note: Groups with same letter superscripts are significantly different at $p<0.05$

$S C A R E D$ screen for child anxiety and related disorders, $S M F Q$ short mood and feelings questionnaire, SNAP-ODD Swanson, Nolan, and Pelham-oppositional defiant disorder subscale, DISC diagnostic interview schedule for children, PTSD post traumatic stress disorder, $M D D$ major depressive disorder, $A D H D$ attention deficit hyperactivity disorder, $O D D$ oppositional defiant disorder, $C D$ conduct disorder

DSM-IV anxiety disorders (Birmaher et al. 1997; Bailey et al. 2006). This score also adequately discriminates between youth who are anxious and those who are depressed or have disruptive behavior disorders, but are not anxious (Birmaher et al. 1999; Monga et al. 2000). The SCARED-P was used to screen youths into the study and to assess anxiety symptom severity.

The Diagnostic Interview Schedule for Children (NIMH DISC-IV; Shaffer et al. 2000) is a structured diagnostic assessment of youth psychopathology with good reliability and validity. The clinic uses a computer-based version of the diagnostic interview completed by the caregiver with the assistance of a trained interviewer. To reduce participant burden, only the modules listed in Table 2 were routinely administered to families, and low-prevalence disorders (e.g., Obsessive Compulsive Disorder) were assessed by clinicians when indicated. The DISC was used in the current study to determine the presence of and impairment associated with an anxiety disorder within the past month and to assess the overall diagnostic profile of each youth. In addition, the DISC provided information about whether youths lived with both parents.

The Short Mood and Feelings Questionnaire parent report (SMFQ-P; Angold et al. 1995) is a 13-item parentreport questionnaire derived from the 34-item mood and feelings questionnaire designed to assess symptoms of depression in youth based on DSM-IV criteria. The SMFQ has correlated highly with other depression self-report measures and has reliably distinguished clinically depressed youth from non-depressed youth. A cutpoint of 11 or more identified the top $6 \%$ of scores in a general youth population (Angold et al. 2002). The SMFQ was used to assess depression symptomatology.

The Swanson, Nolan, and Pelham (SNAP-IV; Swanson 1992) is a 40-item scale measuring the presence and severity of externalizing behaviors with evidence of good 
reliability and validity. In the present study, the score on the ODD subscale was used to assess the extent to which externalizing behavior predicted treatment attrition.

\section{Definition of Dropout}

We adopted a two-part definition of treatment dropout. First, we used a dichotomous dropout variable (yes/no) based on clinician-rated reason for discharge. Next, youths classified as dropouts were further delineated by number of sessions in order to categorize youths who dropped out during different phases of treatment.

\section{Global Clinician-Rated Dropout (CR)}

Upon termination of services, clinicians indicated the reason for discharge in youths' medical record. Subsequently, two raters reviewed these data and classified subjects as dropouts (yes/no) based on the clinician-documented reason for discharge. Dropouts were defined as youths or families who unilaterally decided to terminate treatment and discontinued contact with clinic (e.g., child/family chose to discontinue, unable to follow up with family). Non-dropouts were defined as youths or families who, with their clinician, agreed that presenting problems were solved, symptoms were abated, or terminated for reasons beyond their control (e.g., moving locations, required outside referral). The raters demonstrated good inter-rater reliability $(k=0.89)$. This approach to classifying dropout status is in line with recommendations made by Wierzbicki and Pekarik (1993) and similar to what has been used in previous studies of attrition (e.g., Kendall and Sugarman 1997; Garcia and Weisz 2002; Kazdin and Mazurick 1994; Kazdin and Wassell 1998; Miller et al. 2008).

\section{Phase of Treatment (PT)}

To examine attrition across the course of treatment, we used a revised definition from previous work to further classify youths by time to dropout (Kazdin and Mazurick 1994). Time points were defined as follows: pre-treatment $(0-1$ sessions, where 0 indicates that the intake was completed but treatment was not initiated), early treatment (2-6 sessions), or late treatment ( $\geq 7$ sessions). These time frames were selected for two main reasons. First, the mean length of treatment for empirically tested child anxiety psychosocial interventions is approximately 12 sessions, and 7 or more sessions maps on to completion of roughly half of a probably efficacious treatment dose of child anxiety psychosocial intervention. Second, the cutoff that distinguishes early from late dropout corresponds to those employed by Kazdin and Mazurick (1994) in a sample of externalizing youths, which may provide an interesting point of comparison.
Data Analysis

We implemented a two-step approach to data analysis for each of our definitions of attrition (CR and PT). First, chisquare and $t$-tests were used to compare attrition rates and characteristics of dropouts and non-dropouts. Second, we employed multivariable binomial and multinomial logistic regression models to predict CR and PT dropout using our full a priori set of seven variables (age, gender, minority status, single parent status, and anxiety, depression, and externalizing symptom severity). We planned to retain significant predictors from the full models for follow-up tests in parsimonious post-hoc prediction models. Values for five cases with missing data (on no more than two items) on either the depression or externalizing continuous measures were imputed using the EM algorithm through the ICE function in STATA. Patterns of results were the same for dimensional measures of symptom severity (SCARED, SMFQ, SNAP), and DISC-IV diagnoses for anxiety, depression, and externalizing problems; therefore, dimensional clinical measures were used in all regression analyses to increase power. Due to the small extant youth anxiety attrition literature, we adopted an exploratory approach and a critical alpha was set at 0.05 for all comparisons.

\section{Results}

\section{Attrition}

Table 1 lists demographic characteristics and attrition rates for each definition. By the global CR definition, there were $101(51.3 \%)$ treatment dropouts and 96 non-dropouts (48.7\%). Dropouts attended a median of six sessions. The most commonly cited reason for discharge was "Child/ family chose to discontinue." Non-dropouts attended a median of 14 sessions, and the most often cited reason for discharge was "Services to be provided by another agency." When dividing the 101 CR dropouts by time point of dropout, 25 cases discontinued services pre-treatment, 28 discontinued services in the early treatment phase, and 48 discontinued in the late treatment phase. Overall, treatment dropouts experienced higher levels of depression than non-dropouts, $t_{195}=2.03, p<0.05$, as predicted a priori (see Table 2 ). The early dropout group contained a marginally higher proportion of ethnic minority youth, $\chi^{2}$ $(3, N=197)=6.48, p<0.10$.

Prediction of Dropout

A multivariate logistic regression analysis was used to predict CR dropout status (yes/no) using all seven a priori 
predictors. Only depression symptoms reliably predicted dropout in the presence of all predictor variables $(\mathrm{OR}=$ $1.06,95 \%$ CI: $1.00,1.12, p<0.05)$. Subsequently, depression severity was included to test a final parsimonious model consisting solely of this significant predictor. This model was significant, $\chi^{2}(1, N=197)=4.11$, $p<0.05$, and appeared to be a good fit using the Hosmer and Lemeshow Goodness-of-Fit test, $\chi^{2}(8)=11.871$, $p>0.50$, indicating that prediction of dropout improved when depression symptoms were taken into account. Specifically, in the absence of other variables, the odds of being classified as a dropout reliably increased by a factor of 1.05 (95\% CI: $1.00,1.09, p<0.05$ ), or $5 \%$, for each single-point increase in SMFQ score. In terms of clinical significance, for every increase of one standard deviation in depression symptoms $(S D=6.49)$, the risk of dropout is 1.34 times higher, such that the odds of dropout increased by $34 \%$ for a youth whose SMFQ score was one standard deviation above the mean compared to a youth who had the mean SMFQ score.

To predict PT dropout, multinomial logistic regression was performed through SPSS 16.0 NOMREG. In the presence of all seven a priori variables, minority status was significantly associated with increased odds of attrition in the pre-treatment phase $(\mathrm{OR}=2.78,95 \% \mathrm{CI}: 1.03,7.54)$ and higher depression symptom ratings increased the odds of dropout in the late treatment phase $(\mathrm{OR}=1.08,95 \%$ CI: $1.00,1.16)$. Subsequently, minority status and depression severity were included to test a parsimonious model consisting of these two significant predictors. The parsimonious model was statistically significant, $\chi^{2}(6, N=$ $197)=12.66, p<0.05$, and appeared a good fit to these data using the Deviance test, $\chi^{2}(150)=134.17, p>0.80$, indicating that depression symptoms and minority status alone reliably distinguished non-dropouts from dropouts at different phases of treatment. The odds of dropping out pre-treatment was nearly three times higher for ethnic minorities $(\mathrm{OR}=2.89,95 \% \mathrm{CI}: 1.10,7.60)$, and late treatment dropouts were more likely to report higher levels of depression than non-dropouts $(\mathrm{OR}=1.07,95 \% \mathrm{CI}$ : $1.01,1.13)$. The odds of dropping out late in treatment increased by $7 \%$ for every one-point increase in SMFQ score. In other words, the odds of dropping out for youths in the late treatment phase with an SMFQ score one standard deviation above the mean were 1.54 times (54\%) higher than youths with the mean SMFQ score.

Based on examination of mean rates of dropout by ethnic group (see Table 1), we suspected that the association between minority status and pre-treatment dropout was due to higher attrition of African-American youth. To unpack this relationship, we compared African-American youth to youth from all other ethnic groups. We computed the reduced multinomial model replacing the original minority status variable (yes/no) with a new dummy-coded contrast (African-American/Other). In this model, pretreatment dropouts were three times more likely to be African-American than any other ethnicity $(\mathrm{OR}=3.03$, $95 \%$ CI: $1.16,7.92)$, indicating that ethnicity effects were driven by African-American youths in this sample. Increase in depression symptoms continued to predict late treatment dropout $(\mathrm{OR}=1.08,95 \% \mathrm{CI}: 1.01,1.16)$.

\section{Discussion}

Anxiety is distressing, impairing, chronic, and highly prevalent in youths. However, little is known about these youths in community practice settings and whether youths receive an adequate course of care. To address this gap, this study examined the rates and predictors of attrition among clinically complex youths with significant anxiety symptoms receiving mental health services in an urban community-based OMHC. Attrition in the sample $(51 \%)$ was within the range reported in other outpatient samples (50-75\%; Kazdin 1997); however, this rate was substantially higher than the $23 \%$ rate previously reported for anxious youths in university anxiety clinics and RCTs (Kendall and Sugarman 1997; Pina et al. 2003). Parceling dropout by phase of treatment was useful in identifying predictors that did not emerge when examined by overall dropout (yes/no). In the present study, depression symptom severity and minority status increased risk for attrition, though the action of these predictors varied by definition of attrition. Youth age, gender, single parent status, severity of co-occurring externalizing symptoms, and severity of anxiety did not significantly increase risk for attrition in this sample.

Higher depression symptoms at intake predicted dropout overall, but particularly during the late treatment phase $(\geq 7$ sessions). This is informative given that anxiety and depression in youth, although highly co-occurring (Axelson and Birmaher 2001; Lewinsohn et al. 1998), are often studied separately. These results are consistent with those of previous studies indicating that depression is adversely associated with treatment dropout in children with conduct problems (Luk et al. 2001) and within a general outpatient youth sample (Dierker et al. 2001). Further exploration of mechanisms through which depression influences attrition is warranted. In this sample, depression severity was not significantly related to stressful contextual variables such as single parent status, involvement with child welfare protective services, or receipt of Medicaid.

Our ethnicity results were driven largely by AfricanAmerican youths in the sample, who were three times more likely than youths of other ethnic groups to initiate termination after completing zero or one treatment sessions. This 
corroborates previous findings that ethnic minorities are at greater risk for attrition early in the treatment process (Kazdin and Mazurick 1994; Weersing and Weisz 2002). In this sample, African-American youths were not significantly more depressed than youths of other ethnic groups $\left(t_{195}=1.59, p=0.12\right)$. In previous research that found a significant association between minority status and attrition, African-American youths comprised the largest minority group (Kendall and Sugarman 1997). The second attrition study that did not produce ethnicity effects sampled largely Latino youths (Pina et al. 2003), and it is plausible that sample differences in ethnic composition may have contributed to the contrasting findings of the impact of "minority status." In future work, consideration of specific ethnic groups may be more informative than examination of combined ethnic minority groups. In addition, it would be valuable to unpack cultural and economic variables that may underlie the effect of "ethnicity." For example, minority status is often confounded with socioeconomic disadvantage (Kazdin 1996), and although Medicaid coverage did not predict dropout in any follow-up analyses, significantly more ethnic minorities received Medicaid insurance than Caucasian families in our sample, $\chi^{2}(1, N=195)=15.68, p<0.001$. This investigation was not powered to unravel potential interactions with ethnicity and SES, although larger, diverse community samples typically found in OMHCs may provide a valuable arena for exploring these effects. Notably, in our sample, other stressful contextual factors (e.g., single parent status, child welfare involvement) did not separate African-American youth from youth of other ethnic minority groups, and these factors did not predict attrition.

Present findings extend previous examinations of attrition by studying a representative group of youths who present in community-based mental health settings. These findings are necessarily limited by several constraints of this "real world" setting. In this study, clinician-rated reason for discharge, rated from medical records, was used as the most valid indicator of the intentions of the child and the family for terminating services. However, clinician judgment of reason for discharge is subjective and likely to vary by clinician. Given that services are not time-limited in this OMHC, it is quite possible that some clinicians may tolerate a greater number of missed sessions and keep a case open longer than others. In addition, the phase of treatment definition in the current study may not correspond to the same phase of treatment within other OMHCs and RCTs (e.g., a youth at session 5 in an RCT may be in a very different place in treatment than a youth at session 5 in an OMHC).

The current sample of youths was selected from a broader clinic sample to have clinically significant levels of anxiety using well-validated standardized assessment tools and maps on to other definitions used to identity anxious youths in public service settings (Chavira et al. 2009). Nevertheless, findings must be interpreted with some notable caveats. First, given that many of these youth met criteria for a number of disorders, it may be best to conceptualize these youth as comprising a clinically anxious/ comorbid sample. This is consistent with previous reports that the diagnostic profiles of anxious youths in community-based service settings are complex and have high rates of non-anxiety comorbidity (Chavira et al. 2009; SouthamGerow et al. 2003) and presents a distinct difference from youths typically seen in RCTs. Second, it would be useful to have information regarding the primacy of anxiety symptoms in this sample. If information regarding primary diagnoses were available, it would be interesting to examine associations between attrition and specific anxiety types. The relatively low prevalence of individual anxiety disorders and the high comorbidity within the anxiety disorders (see Table 2) limited our ability to meaningfully examine attrition by anxiety type in this sample or to examine the influence of anxiety alone on attrition. However, such analyses may not be the most informative in this sample given the clinical complexity that characterizes anxious youths in public service settings.

Further, as our data collection was embedded in the daily operation of an active outpatient clinic, our ability to assess certain predictors of attrition was limited by the mission of this setting. This excluded several potentially meaningful predictors of attrition, such as parental diagnoses, which were not systematically assessed as a component of intake into child-based services. In addition, it was difficult to ascertain reliable information regarding medication use. As medication-only services are not provided in this clinic, many youths receive medication prescriptions from pediatricians and providers outside of this clinic prior to, during, and after therapy services. This information may not have been systematically reported to or documented by therapists and findings regarding medication status in this sample would likely be misleading at best. Furthermore, medication use is a complex issue that can vary considerably depending on how medication use is conceptualized (e.g., SSRI vs. non-SSRI vs. no medication; start of medication during treatment, and so on). While we were not in a position to examine the various patterns of medication use, these issues may influence treatment engagement and attendance and warrant future study. Future investigations also would benefit from interviews with providers and families to elucidate process-related reasons for dropout, including perceived barriers to treatment, client-therapist ethnic match, therapeutic alliance, and the pace and content of treatment (Garcia and Weisz 2002; Gross et al. 2001; McCabe 2002; Stevens et al. 2006). 
Implications and Future Directions

There are important service setting characteristics that may have bearing upon interpretation and implications of results. For instance, the rates of non-anxiety psychiatric comorbidity were extremely high in this sample. This profile is consistent with that anxious youths served in public sectors of care, for whom comorbidity rates of approximately $60 \%$ with non-anxiety disorders have been reported (Chavira et al. 2009). Our goal in this investigation was to identify a population who would serve as potential targets for dissemination of youth anxiety treatments as they exist in this outpatient setting. This level of comorbidity has important implications for the treatment planning and engagement process in this outpatient setting. While many of these youths present with a variety of other problems, anxiety is likely an important component of their clinical picture and targeted treatment of anxiety in these youths seems reasonable. In at least one investigation of youths in public settings, significant anxiety in the context of other psychiatric comorbidities was a risk factor for a number of adverse outcomes, including receipt of inpatient treatment (Chavira et al. 2009). In order to better understand factors that may influence treatment and outcomes in this setting, examination of current treatment and course of treatment of youths with significant anxiety is critical.

In addition, mean duration of treatment length varies substantially between research and practice. Youths in OMHCs attend an average of three sessions (Hansen et al. 2002; McKay and Bannon 2004). By contrast, among youth anxiety psychotherapies that meet criteria for "probably efficacious treatment" (see Silverman et al. 2008), the shortest intervention (Barrett et al. 1996) was 12 sessions of CBT. Furthermore, a study examining outcomes after a full course of treatment (12 weeks) from a specialty outpatient clinic for anxiety found that only $43 \%$ of these youths receiving CBT had a "good" treatment response (Southam-Gerow et al. 2001). Notably, less than half $(44 \%)$ of the participants with significant anxiety in the current OMHC sample received 12 sessions or more. If the same $43 \%$ positive response rate were to hold in this sample, only about one in five youths would be expected to have a positive response even if current "best practice" interventions for anxiety were offered. While it is possible that retention may have improved if therapists were providing manualized CBT for anxiety, this remains an open question. A recent study examining the transportability of CBT for depression in a community mental health clinic did not observe significant differences in total missed sessions between youths who received CBT and those who received usual care (Weisz et al. 2009). One recent study has demonstrated that child-focused CBT can be transported to community-based mental health clinics for some anxiety disorders (Farrell et al. 2010); however, as the authors note, the sample was more similar to those seen in traditional RCTs than in typical community mental health practice. A number of recent studies have investigated child anxiety treatment in novel settings, including schools and primary care (e.g., Farrell et al. 2010; Ginsburg et al. 2008; Masia Warner et al. 2007; Weersing et al. 2008), however, several of these child anxiety investigations routinely exclude youths with depressive comorbidity. Indeed, of 32 studies included in a recent review of evidence-based treatments for child phobia and anxiety (Silverman et al. 2008), at least six (19\%) excluded youths with depression (three did not provide this information). Of the 23 studies in which depression was not an exclusion criterion, eight did not enroll any depressed youth, five did not provide rates of depression, and the remaining studies included a small proportion (average of $8.5 \%$ of sample) of concurrently depressed youth. Inclusion of youths with elevated levels of depression may help mollify concerns that findings from research clinics may not generalize to other service settings, especially given our provocative data that this common comorbidity may substantially increase the risk of treatment dropout.

Present findings also suggest the potential value of research aimed at improving response and increasing family engagement very early in treatment. Our results indicate that African-American youths are at risk for dropping out within the first few sessions, which merits additional, targeted efforts to engage these youths and their families at treatment initiation. It also is plausible that one reason for early attrition is lack of quick clinical improvement. For more clinically severe youths, converging data show that combination treatments (i.e., CBT and SSRI) may be efficacious in maximizing speed of response for both anxiety and depression (Walkup et al. 2008). In addition, internalizing youths may benefit from integrated psychosocial treatments designed to target both anxiety and depression to produce a quick and robust response (Trosper et al. 2009; Weersing et al. 2008). Broadly, speed of response may be critical for provision of services in usual care settings given that many youths attend treatment for short periods and underscores the importance of attrition prevention early in treatment. Further examination of whether rapid improvement in internalizing symptoms increases retention, and factors that influence response rate (e.g., medication use), is warranted.

Broadly speaking, we see value in developing a more complete picture of patterns of service use among clinically and demographically diverse youths for whom anxiety and depression present significant difficulties. Specifically, identification of predictors of treatment attrition may lead to fruitful efforts to develop interventions and pre-treatment or in-session strategies that may mitigate the impact of these 
variables. Such techniques may help bridge the gap between research and practice by increasing the "robustness" of treatment and facilitating dissemination efforts.

Open Access This article is distributed under the terms of the Creative Commons Attribution Noncommercial License which permits any noncommercial use, distribution, and reproduction in any medium, provided the original author(s) and source are credited.

\section{References}

Angold, A., Costello, E. J., Messer, S. C., \& Pickles, A. (1995). Development of a short questionnaire for use in epidemiological studies of depression in children and adolescents. International Journal of Methods in Psychiatric Research, 5, 237-249.

Angold, A., Erkanli, A., Silberg, J., Eaves, L., \& Costello, J. (2002). Depression scale scores in 8-17 year-olds. Journal of Child Psychology and Psychiatry, 43, 1052-1063.

Armbruster, P., \& Kazdin, A. E. (1994). Attrition in child psychotherapy. Advances in Clinical Child Psychology, 16, 81-108.

Attride-Stirling, J., Davis, H., Farrell, L., Groark, C., \& Day, C. (2004). Factors influencing parental engagement in a community child and adolescent mental health service: A qualitative comparison of completers and non-completers. Clinical Child Psychology and Psychiatry, 9, 347-361.

Axelson, D. A., \& Birmaher, B. (2001). Relation between anxiety and depressive disorders in childhood and adolescence. Depression and Anxiety, 14, 67-78.

Bailey, K. A., Chavira, D. A., Stein, M., \& Stein, M. (2006). Brief measures to screen for social phobia in primary care pediatrics. Journal of Pediatric Psychology, 5, 513-521.

Barrett, P. M., Dadds, M. R., \& Rapee, R. M. (1996). Family treatment of childhood anxiety: A controlled trial. Journal of Consulting and Clinical Psychology, 64, 333-342.

Birmaher, B., Khetarpal, S., Brent, D., Cully, M., Balach, L., Kaufman, J., et al. (1997). The screen for child anxiety related emotional disorders (SCARED): Scale construction and psychometric characteristics. Journal of the American Academy of Child and Adolescent Psychiatry, 36, 545-553.

Birmaher, B., Brent, D. A., Chiappetta, L., Bridge, J., Monga, S., \& Baugher, M. (1999). Psychometric properties of the screen for child anxiety related emotional disorders (SCARED): A replication study. Journal of the American Academy of Child and Adolescent Psychiatry, 38, 1230-1236.

Bittner, A., Egger, H. L., Erkanli, A., Costello, E. J., Foley, D. L., \& Angold, A. (2007). What do childhood anxiety disorders predict? Journal of Child Psychology and Psychiatry, 48, 1174-1183.

Cartwright-Hatton, S., McNicol, K., \& Doubleday, E. (2006). Anxiety in a neglected population: Prevalence of anxiety disorders in preadolescent children. Clinical Psychology Review, 26, 817-833.

Chavira, D. A., Garland, A., Yeh, M., McCabe, K., \& Hough, R. L. (2009). Child anxiety disorders in public systems of care: Comorbidity and service utilization. Journal of Behavioral Health Services and Research, 36, 492-504.

Compton, S. N., March, J. S., Brent, D., Albano, A. M., Weersing, V. R., \& Curry, J. (2004). Cognitive-behavioral psychotherapy for anxiety and depressive disorders in children and adolescents: An evidence-based medicine review. Journal of the American Academy of Child and Adolescent Psychiatry, 43, 930-959.

Costello, J. E., Mustillo, S., Erkanli, A., Keeler, G., \& Angold, A. (2003). Prevalence and development of psychiatric disorders in childhood and adolescence. Archives of General Psychiatry, 8, 837-844.
Dadds, M. R., Holland, D. E., Laurens, K. R., Mullins, M., Barrett, P. M., \& Spence, S. H. (1999). Early intervention and prevention of anxiety disorders in children: Results at 2-year follow-up. Journal of Consulting and Clinical Psychology, 67, 145-150.

Dierker, L., Nargiso, J., Wiseman, R., \& Hoff, D. (2001). Factors predicting attrition within a community initiated system of care. Journal of Child and Family Studies, 10, 367-383.

Emslie, G. J. (2008). Pediatric anxiety-Underrecognized and undertreatead. The New England Journal of Medicine, 359, 28352836.

Farrell, L. J., Schlup, B., \& Boschen, M. J. (2010). Cognitivebehavioral treatment of childhood obsessive-compulsive disorder in community-based clinical practice: Clinical significance and benchmarking against efficacy. Behaviour Research and Therapy, 48, 409-417.

Friars, P., \& Mellor, D. (2009). Drop-out from parenting training programmes: A retrospective study. Journal of Child and Adolescent Mental Health, 21, 29-38.

Garcia, J. A., \& Weisz, J. R. (2002). When youth mental health care stops: Therapeutic relationship problems and other reasons for ending youth outpatient treatment. Journal of Consulting and Clinical Psychology, 70, 439-443.

Garfield, S. L. (1994). Research on client variables in psychotherapy. In A. E. Bergin \& S. L. (Eds.), Handbook of psychotherapy and behavior change (4th ed.), (pp. 190-228).

Ginsburg, G. S., Becker, K. D., Kingery, J. N., \& Nichols, T. (2008). Transporting CBT for childhood anxiety disorders into inner-city school-based mental health clinics. Cognitive and Behavioral Practice, 15, 148-158.

Gross, D., Julion, W., \& Fogg, L. (2001). What motivates participation and dropout among low-income urban families of color in a prevention intervention? Family Relations, 50, 246-254.

Hansen, N. B., Lambert, M. J., \& Forman, E. M. (2002). The psychotherapy dose-response effect and its implications for treatment delivery services. Clinical Psychology: Science and Practice, 9, 329-343.

Harpaz-Rotem, I., Leslie, D., \& Rosenheck, R. A. (2004). Treatment retention among children entering a new episode of mental health care. Psychiatric Services, 55(9), 1022-1028.

Hunt, C., \& Andrews, G. (1992). Drop-out rate as a performance indicator in psychotherapy. Acta Psychiatrica Scandinavica, 85, $275-278$.

Kashani, J. H., \& Orvaschel, H. (1990). A community study of anxiety in children and adolescents. American Journal of Psychiatry, 147, 313-318.

Kazdin, A. E. (1990). Premature termination from treatment among children referred for antisocial behavior. Journal of Consulting and Clinical Psychology, 31, 415-425.

Kazdin, A. E. (1996). Combined and multimodal treatments in child and adolescent psychotherapy: Issues, challenges, and research directions. Clinical Psychology-Science \& Practice, 3, 69-100.

Kazdin, A. E. (1997). A model for developing effective treatments: Progression and interplay of theory, research, and practice. Journal of Clinical Child Psychology, 26, 114-129.

Kazdin, A. E., \& Mazurick, J. L. (1994). Dropping out of child psychotherapy: Distinguishing early and late dropouts over the course of treatment. Journal of Consulting and Clinical Psychology, 62, 1069-1074.

Kazdin, A. E., \& Wassell, G. (1998). Treatment completion and therapeutic change among children referred for outpatient therapy. Professional Psychology-Research \& Practice, 29, 332-340.

Kazdin, A. E., Mazurick, J. L., \& Bass, D. (1993). Risk for attrition in treatment of antisocial children and families. Journal of Clinical Child Psychology, 22, 2-16.

Keller, M. B., Lavori, P. W., Wunder, J., Beardslee, W. R., Schwartz, C. E., \& Roth, J. (1992). Chronic course of anxiety disorders in 
children and adolescents. Journal of the American Academy of Child and Adolescent Psychiatry, 31, 595-599.

Kendall, P. C., \& Sugarman, A. (1997). Attrition in the treatment of childhood anxiety disorders. Journal of Consulting and Clinical Psychology, 65, 883-888.

La Greca, A. M., Silverman, W. K., \& Lochman, J. E. (2009). Moving beyond efficacy and effectiveness in child and adolescent intervention research. Journal of Consulting and Clinical Psychology, 77, 373-382.

Lewinsohn, P. W., Rohde, P., \& Seeley, J. R. (1998). Major depressive disorder in older adolescents: Prevalence, risk factors, and clinical implications. Clinical Psychology Review, 18, 765-794.

Luk, E. S. L., Staiger, P. K., Mathai, J., Wong, L., Birleson, P., \& Adler, R. (2001). Children with persistent conduct problems who dropout of treatment. European Child and Adolescent Psychiatry, 10, 28-36.

Masia Warner, C., Fisher, P. H., Shrout, P. E., Rathor, S., \& Klein, R. G. (2007). Treating adolescents with social anxiety disorder in school: An attention control trial. Journal of Child Psychology and Psychiatry, 48, 676-686.

May, D. E., Kratochvil, C. J., Puumala, S. E., Silva, S. G., Rezac, A., Hallin, M. J., et al. (2007). A manual-based intervention to address clinical crises and retain patients in the Treatment of Adolescents with Depression Study (TADS). Journal of the American Academy of Child and Adolescent Psychiatry, 46, 573-581.

McCabe, K. M. (2002). Factors that predict premature termination among Mexican-American children in outpatient psychotherapy. Journal of Child and Family Studies, 11, 347-359.

McKay, M. M., \& Bannon, W. M. (2004). Engaging families in child mental health services. Child and Adolescent Psychiatric Clinics of North America, 13, 905-921.

McKay, M., Stoewe, J., McCadam, K., \& Gonzales, J. (1998). Increasing access to child mental health services for urban children and their caregivers. Health and Social Work, 23(1998), 9-15.

Miller, L. M., Southam-Gerow, M. A., \& Allin, R. B. (2008). Who stays in treatment? Child and family predictors of youth client retention in a public mental health agency. Child \& Youth Care Forum, 37, 153-170.

Monga, S., Birmaher, B., Chiappetta, L., Brent, D., Kaufman, J., Bridge, J., et al. (2000). Screen for Child Anxiety-Related Emotional Disorders (SCARED): Convergent and divergent validity. Depression and Anxiety, 12, 85-91.

Nock, M. K., \& Kazdin, A. E. (2005). Randomized controlled trial of a brief intervention for increasing participation in parent management training. Journal of Consulting and Clinical Psychology, 73, 872-879.

Ollendick, T. H., Jarrett, M. A., Grills-Taquechel, A. E., Hovey, L. D., \& Wolff, J. C. (2008). Comorbidity as a predictor and moderator of treatment outcomes in youth with anxiety, affective, attention deficit/hyperactivity disorder, and oppositional/conduct disorders. Clinical Psychology Review, 28, 1447-1471.

Pelkonen, M., Marttunen, M., Laippala, P., \& Lannqvist, J. (2000). Factors associated with early dropout from adolescent psychiatric outpatient treatment. Journal of the American Academy of Child \& Adolescent Psychiatry, 39, 329-336.

Pina, A. A., Silverman, W. K., Weems, C. F., Kurtines, W. M., \& Goldman, M. L. (2003). A comparison of completers and noncompleters of exposure-based cognitive and behavioral treatment for phobic and anxiety disorders in youth. Journal of Consulting and Clinical Psychology, 71, 701-705.

Sayal, K., Taylor, E., Beecham, J., \& Byrne, P. (2002). Pathways to care in children at risk of attention-deficit hyperactivity disorder. British Journal of Psychiatry, 181, 43-48.
Shaffer, D., Fisher, P., Lucas, C. P., Dulcan, M. K., \& Schwab-Stone, M. E. (2000). NIMH diagnostic interview schedule for children version IV (NIMH DISC-IV): Description, differences from previous versions, and reliability of some common diagnoses. Journal of the American Academy of Child and Adolescent Psychiatry, 39, 28-38.

Silverman, W. K., Pina, A. A., \& Viswesvaran, C. (2008). Evidencebased psychosocial treatments for phobic and anxiety disorders in children and adolescents. Journal of Clinical Child and Adolescent Psychology, 37, 105-130.

Sledge, W. H., Moras, K., Hartley, D., \& Levine, M. (1990). Effect of time-limited psychotherapy on patient dropout rates. American Journal of Psychiatry, 147, 1341-1347.

Southam-Gerow, M. A., Kendall, P. C., \& Weersing, V. R. (2001). Examining outcome variability: Correlates of treatment response in a child and adolescent anxiety clinic. Journal of Clinical Child Psychology, 30, 422-436.

Southam-Gerow, M. A., Weisz, J. R., \& Kendall, P. C. (2003). Youth with anxiety disorders in research and service clinics: Examining client differences and similarities. Journal of Clinical Child \& Adolescent Psychology, 32, 375-385.

Stevens, J., Kelleher, K. J., Ward-Estes, J., \& Hayes, J. (2006). Perceived barriers to treatment and psychotherapy attendance in child community mental health centers. Community Mental Health Journal, 42, 449-458.

Swanson, J. M. (1992). School-based assessments and interventions for ADD students. Irvine, CA: K. C. Publishing.

Trosper, S. E., Buzzella, B. A., Bennett, S. M., \& Ehrenreich, J. T. (2009). Emotion regulation in youth with emotional disorders: Implications for a unified treatment. Clinical Child and Family Psychology Review, 12, 234-254.

Walitzer, K., Dermen, K., \& Conners, G. (1999). Strategies for preparing clients for treatment: A review. Behavior Modification, 23, 129-151.

Walker, P. N., Weersing, V. R., Warnick, E. M., Findley, D., Scahill, L., \& Woolston, J. (2008). Predictors of youth therapist technique use in community care. Orlando, Florida: Poster presented at the annual meeting of the Association for Behavioral and Cognitive Therapies.

Walkup, J. T., Albano, A. M., Piacentini, J., Birmaher, B., Compton, S. N., Sherrill, J. T., et al. (2008). Cognitive behavior therapy, sertraline, or a combination in childhood anxiety. The New England Journal of Medicine, 359, 2753-2766.

Weersing, V. R., \& Weisz, J. R. (2002). Community clinic treatment of depressed youth: Benchmarking usual care against CBT clinical trials. Journal of Consulting and Clinical Psychology, 70, 299-310.

Weersing, V. R., Gonzalez, A., Campo, J., \& Lucas, A. (2008). Brief behavioral therapy for pediatric anxiety and depression: Piloting an integrated treatment approach. Cognitive and Behavioral Practice, 15, 126-139.

Weisz, J. R., Weiss, B., \& Donenberg, G. R. (1992). The lab versus the clinic: Effects of child and adolescent psychotherapy. American Psychologist, 47, 1578-1585.

Weisz, J. R., Donenberg, G. R., Han, S. S., \& Weiss, B. (1995a). Bridging the gap between laboratory and clinic in child and adolescent psychotherapy. Journal of Consulting and Clinical Psychology, 63, 688-701.

Weisz, J. R., Donenberg, G. R., Han, S. S., \& Kauneckis, D. (1995b). Child and adolescent psychotherapy outcomes in experiments versus clinics: Why the disparity? Journal of Abnormal Child Psychology, 23, 83-106.

Weisz, J. R., Southam-Gerow, M. A., Gordis, E. B., Connor-Smith, J. K., Chu, B. C., Langer, D. A., et al. (2009). Cognitive-behavioral therapy versus usual care for youth depression: An initial test of 
transportability to community clinics and clinicians. Journal of Consulting and Clinical Psychology, 77, 383-396.

Wierzbicki, M., \& Pekarik, G. (1993). A meta-analysis of psychotherapy dropout. Professional Psychology: Research and Practice, 24, 190-195.

Woodward, L. J., \& Fergusson, D. M. (2001). Life course outcomes of young people with anxiety disorders in adolescence. Journal of the American Academy of Child and Adolescent Psychiatry, 40, 1086-1093.

Wu, P., Hoven, C. W., Bird, H. R., Moore, R. E., Cohen, P., Alegria, M., et al. (1999). Depressive and disruptive disorders and mental health service utilization in children and adolescents. Journal of the American Academy of Child and Adolescent Psychiatry, 38, 1081-1090. 